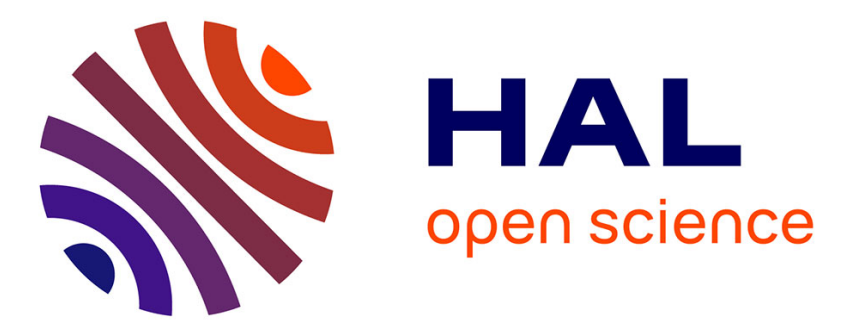

\title{
Infrastructures et oasis-relais migratoires au Sahara algérien
}

Jacques Fontaine

\section{To cite this version:}

Jacques Fontaine. Infrastructures et oasis-relais migratoires au Sahara algérien. Annales de géographie, 2005, 644, pp.437-448. 10.3406/geo.2005.21256 . hal-02502819

\section{HAL Id: hal-02502819 \\ https://u-bourgogne.hal.science/hal-02502819}

Submitted on 9 Mar 2020

HAL is a multi-disciplinary open access archive for the deposit and dissemination of scientific research documents, whether they are published or not. The documents may come from teaching and research institutions in France or abroad, or from public or private research centers.
L'archive ouverte pluridisciplinaire HAL, est destinée au dépôt et à la diffusion de documents scientifiques de niveau recherche, publiés ou non, émanant des établissements d'enseignement et de recherche français ou étrangers, des laboratoires publics ou privés. 


\section{Infrastructures et oasis-relais migratoires au Sahara algérien}

\section{Jacques Fontaine}

\section{Citer ce document / Cite this document :}

Fontaine Jacques. Infrastructures et oasis-relais migratoires au Sahara algérien. In: Annales de Géographie, t. 114, $n^{\circ} 644,2005$. pp. 437-448;

doi : https://doi.org/10.3406/geo.2005.21256

https://www.persee.fr/doc/geo_0003-4010_2005_num_114_644_21256

Fichier pdf généré le 24/02/2020 


\title{
Infrastructures et oasis-relais migratoires au Sahara algérien
}

\author{
Algerian Salhara migratory oasis-relay networks \\ Jacques Fontaine \\ Maitre de Contérences, Universilé de Franche-Conté \\ THLAMA (Theoriser et modètiser pour aménager), CNRS-UAR 6049, Besancon
}

L'immensité est une donnée essentielle du Sahara. Avec plus de deux millions de $k^{2}$ et moins de trois millions d'habitants, te Sahara algérien est un espace particulier où la présence de l'homme, attestée depuis des millénaires, est toujours en situation fragile et où ses déplacements ont longtemps été risqués... Ils le sont partois encore. Les caravanes ont relié pendant des centaines d'années les oasis entre elles et arec les zones de pâturages, ainsi qu'avec les espaces bordiers du désert (Maghreb au nord, Sahel au sud).

C'est seulement après la Seconde Guerre mondiale que la France s'est intéressée au désenclavement du Sahara algérien. En une quinzaine d'années routes et pistes d'atterrissage ont été implantées en plein déserr... pour les besoins des pétroliers et des militares. Après 1962, l'Algérie indépendante s'est lancéc dans un effort remarquable, pour l'accès aux ressonices en hydrocinbures, pour le déscnclavenuent des populations oasiennes... et pour le contrôle de cet immense désert parfois convoité. Aujourd'hui, les infrastructures de communication ont été largement anéliorées encore quatucune route ne traverse entièrement le Sahara du nord au sud. Elles mettent en relation des oasis dont le rôle de pôles d'échanges et de relais migratoire ne fait que croître.

Utilisant les nouvelles infrastructures et les oasis relais, les migrations de population se sont développées et complexifiées: aux anciennes migrations intra-sahariennes et Sahara-Tell se sont ajoutées les migrations de travail ou définitives de l'Algéric du Nord vers les chantiers d'hydrocarbures, les infrastructures administratives et, parfois, vers les nouvelles mises en valeur agricoles.

\section{$1 \quad$ Un désert de plus en plus ouvert}

\subsection{Des voies ferrées sans lendemain}

Pendant la plus grande partie de la période coloniale la France, qui s’intéressait peu au Sahara (Bisson, 1996) nª pas cherché à le désenclaver. Elle 
pensa d'abord à installer des voies ferrées (à écartenent métrique) de AïnSefra à Bechar, de lBiskra à Touggourt et El-Oued et jusqu'à Laghouar (ce dernier projer ne tut pas réalisé). Entre les deux guerres fut lancé, à partir du Maroc er via Bechar, le faneux projet "Méditerranée-Niger" qui devait permettre de relies l'Afrique occidentale française à l'Afrique du Nord et, au-delà, à la France. Les promoteurs de ce projet persaient amener ainsi en métropole les richesses de l'Afrique et surtout, y déverser les produits de l'industrie française. Las, vicume de la crise des années trente, puis de la Seconde Guerre mondiale et enfin de la concurrence de l'automobile, du camion et de l'avion, la voie ferrée ne dépassa Bechar que de quelques dizaines de km!

Aujourd'hui le réseau ferré saharien est des plus squelettiques, seule la voie Biskra/louggourt, modemisée et mise à l'écartement normal à la fin des années cinquante pour les besoins du gisement pétrolier d'I-Hassi-Messaond a cncore un faible trafic voyageurs et marchandises. La voie vers ElOued a été déposée, de même que les quelques dizaines de km au-delà de Bechar. Quant à la voie de Bechar elle n'est utilisée que pour les marchandises. Cependant, il semble que les autorités algériemnes s'intéressent à nouveau à la voie ferrée. Le prolongenant de la voie de Touggourt vers Hassi-Messaoud et Ouargla est de nouveau évoqué, ainsi qu'une très éventuelle poursuite vers Ghardaïa et Laghouat, puis Djelfa afin de rejoindre une voie existante. Plus concrètement, un appel d'offres a été lancé durant l'éré 2003 pour la modernisation et la mise à l'écartement normal de la voie de Bechar, ce qui permetta une meilleure connexion au reste du réseau ex une relance du trafic.

\subsection{Une desserte routière étendue dans la seconde moitié $d u x x^{p e}$ siècle}

\subsubsection{Trois portes, trois pénétrantes sahariennes}

L'entrée au Sahara se faic essenciellement par trois «portes» situées dans 1"Atlas saharien ou sur sa bordure méridionale. Elles s'ouvent sur trois axes essentiels tracés en direction du centre du désert. Ces axes épousent les contraintes du milieu naturel. Its bénéficient de la présence de relais, les oasis. Trois jouent à ce titre un ròle essentiel.

Ain-Sefrar, au coenr des Monts des Ksour, est la prenaère oasis rencontrée quand on vient d'Oran ou de Tlemcen; elle marque le départ via Bechar, de la vieille piste qui reliait dès l'époque médiévale le Maghreb et le Sahel en empruntant la vallée de la Saoura et le Touat. Elle est jalonnée par un chapelet d'oasis avant de traverser l'inhospitalier Tanczrouft (le pays du vide en tamacheg) pour atteindre Gao er Tombouctou.

Laghouat, an pied des Ouled Naïl est sur le même méridien qu'Alger; alle permet de rejoindre la pentapole mozabite (Gliardaïa), puis El-Meniaa, In-Salah er le Tidikelt, le Hoggar (Tamanghasset) et enfin Agadez et l'Aïr, puis Zinder au cour du Bilad as-Sudan.

Biskra, au pied des Aurès, est la porte orientale du Saliara algérien. Ce troisieme axe n'avait autrefois pas l'importance des deux premiers, mais il 
dessert aujourd'hui les principales régions oasiennes: le Souf (El-Oued), l'Oued-Righ (Touggourt), Ouargla, ainsi que les régions pétrolières. Audelà, soit il rejoint El-Menia, soit il s'enfonce vers le sud-est à travers un couloir interdunaire du Grand Ero oriental (le Gassi Touil) en direction du Tassili n'Ajjer et de Djanet. De là, il gagne à travers le 'Ténéré, soit l'Aïr, soit le Kawar et les pays du 'Tchad.

\subsubsection{Un désenclavement progressif du Sahara profond}

C'est seulement après la Seconde Guerre mondiale qu'un effort a été fait pour créer des routes modernes qui ont pernis, très progressivement, un désenclavenent au moins particl des immensités sahariennes.

Si des travaux d'entretien des pistes étaient faits dès la première moitié du $x^{\circ}$ siècle, la création de routes a commencé seulement après 1945. En 1956, seuls deux tronçons représentant à peine plus de $400 \mathrm{~km}$ étaient goudronnés: Biskra/Touggourt (RN 3) et Laghourat/Ghardaïa (RN 1). En 1960, Bechar était desservie depuis Ain-Sefia, El-Menia depuis Ghardaĩa, El-Oued était jointe à partir de la route de Touggourt; mais le plus important était la double liaison vers Ouargla à partir de Ghardaia à l'ouest et de 'Touggourt au nord, prolongée vers Hassi-Messaoud et au-delà jusqu'ì Fort-Flatters (aujourd'hui Hassi-bel-Guebbour), en direction d'Edjeleh. En quatre ans plus de $1100 \mathrm{~km}$ de routes bitumées avaient été réalisées. Les découvertes de pétrole à Edjeleh et Hassi-Messaoud n'y étaient pas pour lien (fig. 1).

La première décentaie de l'inctépendance fut marquée par la poursuite de l'équipement des zones pétrolières surtout dans le secteur. d'In-Aménas. Le désenclavement des oasis de l'ouest (Saoura, Touat, Gourara), par la créarion de la route méridienne Béclnar/Adrar et de la transversale ElMeniaa/Timimoun/Adrar fut également recherché; ce fut aussi l'époque de l'amélioration de la desserte du Bas-Sahara par la mise en service de la route Tebessa/El-Oued/Touggourt et de plusieurs petites antennes dans les régions de Biskra, Glıardaïa, lagghouat... Enfin, au lendemain de la «guerre des sables» (1963) au cours de laquelle le Maroc centa de «récupérer la portion du Sahara qui lui revenait» (selon le régime chérifien), et son échec, l'Algérie a entrepris la création de la route suatégique vers Tindouf ( $800 \mathrm{~km}$ au-delà de Bechar). Ainsi, en 1971, l'essentiel du Sahara utile (zones pétrolières, grandes régions d'oasis) était désenclavé. Seuls le Tidikelt et le grand sud (Tamanghasser, Djanet) restaient à l'écart du bitume.

Le grand ouvre des années 1970 fut la réalisation de la transsaharienne, ou route de l'Unité africane. Elle devait joindre la Méditeranée à l'Afrique. noire, réactivant ainsi un vieil axe méridien qui avait fortenent décliné pendant la colonisation (Blin, 1990). Ce projet, considérable, relia dans un premier temps El-Menia à In-Salah $(400 \mathrm{~km})$ puis ultérieurement à Tamanglaasset $(650 \mathrm{~km})$. Ce second tronçon, inauguré en 1978 , connut des difficultés de réalisation à cause de l'hostilité du milieu, de la rareté des points d'eau et de l'absence d'oasis entre In-Salah et la capitale du Hoggar. 


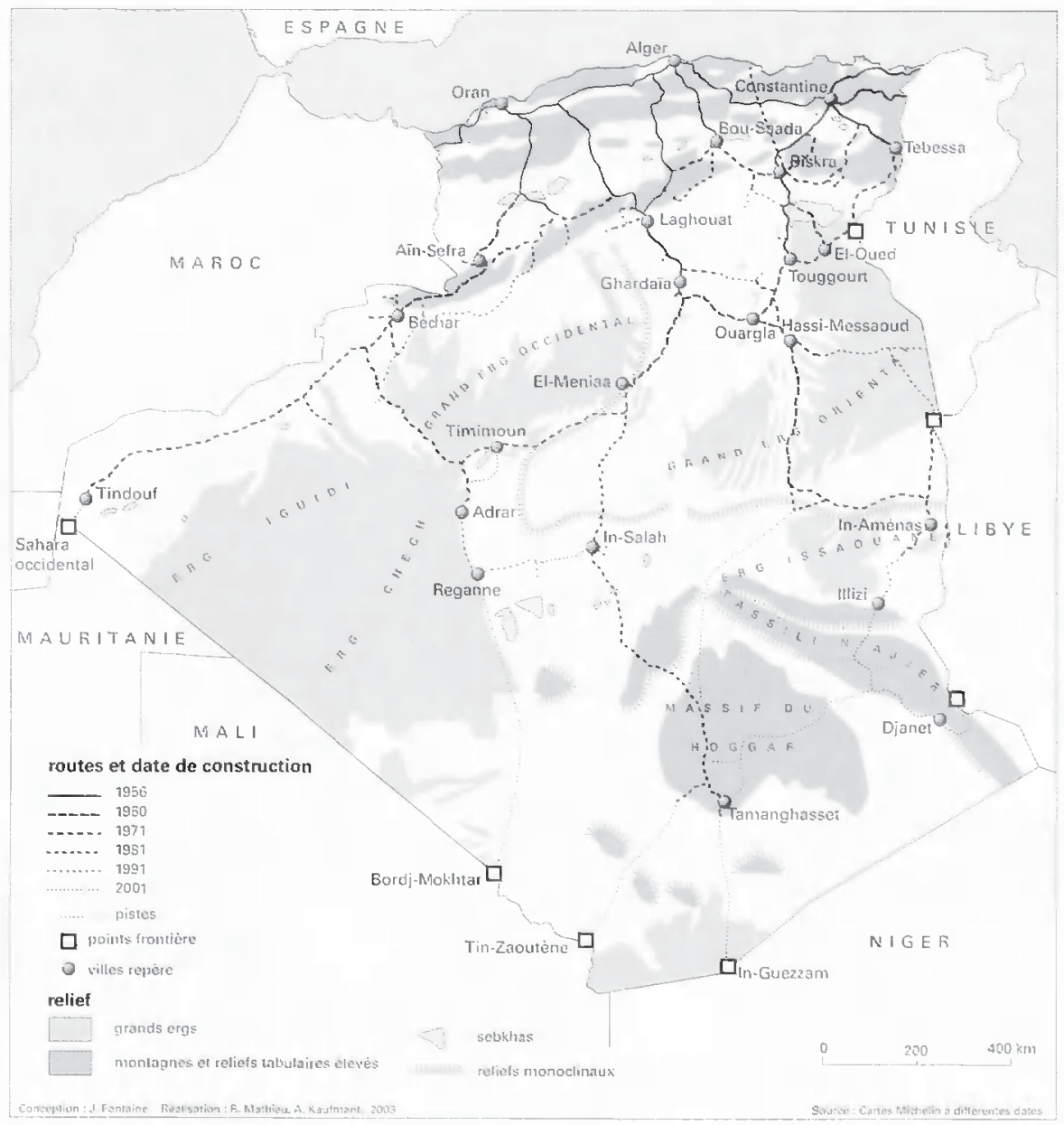

Fig. 1 Evolution du réseau routier du Sahara algérien.

Development of the Algerian Sahara road network.

Ces difficultés entrainèrent le report des deux branches méridionales de la transsaharienne, vers Gao (Mali) via Tin-Zaoutène et Kidal, vers Agadès (Niger) via In-Guczzam et Arlit. Seuls quelques dizaines de km, permettant de désenclaver quelques villages tonareg furent réalisés dans les anuécs 1980 autour de Tamanghasser.

Les années 1980 n'ont pas vu d'opération aussi spectaculaires. Elles furent marquées par l'ouverture d'une route El-Abiod-Sidi-Cheikh/ Taghit/Igli, parallèle à la nationate 6, Aün-Sefra/Bechar/Saoula. Cene dernière présentant l'inconvénient de passer à quelques $\mathrm{km}$ de la frontière marocaine, on peut done supposer que la préoccupation stratégique ne fut: pas érangère à la réalisation de la nouvelle route. Le désenclavenent du 
sud du Touat (Reggane) et de l'ouest du Tidikelt (Aoulef), celui de Illizi, seul chef-lieu de wilaya non encore raccordé au réseau routier national sont également à mettre au crédit de cette décemie, de même que la création d'une route dans le Grand Erg oriental afin de desservir le petit gisement pétrolier frontalier d'El-Borma et de raccourcir la distance vers Ghadamès (Libye). Mais construire une route en plein erg est un travail sans espoir, équivalent au mythe de Sisyphe, et quelques ammées après, la route était revenue à l'état de piste. En outre, comme dans la période précédente, quelques antennes ou quelques liaisons entre oasis (telle El-Alia/Guerrara) futent réalisées.

La dernière période n'a vu qu"une réalisation importante. Il s'agit de la liaison 1llizi/Djanet à travers le Tassili n'Ajjer. On peut aussi noter la construction d'une seconde liaison Timimoun/Adrar desservant les oasis de l'Aougrout ainsi que la mise en service complète de la route Aoulef/InSalah à travers le Tidikelt. Elle raccorde par un second itinéraire l'axe occidental (Saoura/Tourt/Tanezrouft) à l'axe central (Mzab/Hoggar)

Ainsi, alor's qu'au lendemain de la Seconde Guerre mondiale il n'y avait que des pistes, aujourd'hui le Sahara algérien est parcouru par plus de $8000 \mathrm{~km}$ de routes revêtues dont plus de 6500 ont été construits après l'indépendance. Désormais, toutes les oasis importantes (Djanet fut la dernière en 2001) sont reliées au réseau routier, ansi que la plus grande partie des chefs-lieux de communes sahariennes. On note cependant quelques exceptions: les chefs-lieux des deux communes montagneuses du Hoggar, ainsi que les chefs-lieux des quatre communes les plus méridionales du pays, aux frontières du Niger et du Mali. De plus, de nombreux petits villages, en particulier dans le Hoggar et le Tassili (Amgurid, Tamadjert, Afara), restent très éloignés du réseau routier. En revanche, si des routes permettent de se rendre dans le Sahara tunisien et libyen, les comnexions sont toujours impossibles avec le Maroc (pour des raisons politiques). Elles ne se font que par la piste avec les pays du Sahel. Néammoins, cette situation devait changer prochainement, le plan de relance économique de 2001 prévoit des crédits importants pour les infrastuctures routières sahariennes; trois axes seraient concernés: Reggane/Bordj-Mokhtar frontière malienne, Tamanghasset/In-Guezzam frontière nigérienne, Djanet/ Tin-Alkoum frontière libyenne. Ainsi, plus de trente ans après son lancement, la transsaharienne serait enfin réalisée.

\subsubsection{Des oasis en chapelets, toutes desservies}

Le Sahara algérien est aujourd'hui bien relié à l'Algérie du Nord et intégré à l'espace national. Cependant on peut opposer trois régions: Sahara du nord, Sahara central et Sahara méridional.

Le Sahara du nord est quadrillé par un réseau routier assez important ol̀ radiales et transversales se complètent pour permettre une bonne accessibilité aux nombreuses oasis. Celles du Bas-Sahara (Ziban, Souf, OuedRigh, Ouargla, Hassi-Messaoud), les plus importantes du point de vue économique et les plus peuplées, sont les mieux reliées à l'Algérie du Nord 
(cing routes différentes joignent les Ziban aux Hautes l'laines et au Tell). Un véritable maillage met en connexion les diverses régions oasiennes et, à l'intérieur de chaque région, les différentes oasis; les plus isolées sont desservies en antenne. Le piémont central de l'Atlas Saharien (Laghouat. Mzab) bénéficie lui aussi, d'une bonne couverture grâce à la route nationale $n^{\circ} 1$, à ses liaisons vers le Sud et l'Est et à de multiples antennes. Quant au centre nord du Salhara, la desserte y est nettement moins fine, a l'exception de la région de Béchar. Le Grand Erg occidental n'est pas traversé mais contourné par les nationales, 1 et 6 , reliées par une uransversale.

Le Sahara central est marqué par quatre pénétrantes Nord-Sud, généralement isolées les unes des autres. A l'exception du Touat et du Tidikelt, il $n^{2} y$ a pas ici de grandes régions d'oasis, mais seulement des villes relais ayant une fonction d'étape vers le Sud. Il n'y a plus ici de résenu maillé, mais seulement une desserte minimale complétée par des pistes. D\%ouest en est, ces quatre pénétrantes donnent accès à quatre destinations: "Tindouf, les camps des réfugiées sahraouis et la Mauritanie; le Touat puis, par une longue piste, Bordj-Moklitar et le Mali; Tamanghasset et le Hoggar, via In-Salah; Djanet et le Tassili n'Ajjer, via Illizi. A l'exception des axes cenuaux reliés par la route (récemment terminée) Reggane/ln-Salah, ces pénétrantes nont pas le lien entre elles. À l'ouest, un immense vide, occupé par les erge lguidi et Chech, sépare les denx axes.

Enfin le Sahara méridional ne dispose d'aucunc route, le goudron dépassant à peine Tamangluasset. Cependant plusieurs pistes convergent vers cette ville qui est le premier centre saharien d'immigrants originaires de l'étranger. Cette inmigration actuclle, essentiellement d'origine sahélienne, est la suite logique des mouvements migratoires liés aux sécheresses des anmées 1980 et aux guerres des années 1990. Hls ont entraîné la création de foyers de peuplement à la fromvière algérienne (Bordj-Mokhtar, Timiaouine, Tin-Zaoutène, In-Guezzann), tous plus ou moins bien retiés par piste à Tamangliasset.

\subsection{Le développement du transport aérien}

Si quelques avions se sont posés au Sahara avant la Seconde Guerre mondiale, ils ont été fort peu nombreux. Ce n'est qu'au terme du conflit que furent installées les premières pistes d'atterrissage à Biskra en 1946 et ì Bechar en 1950 (fig. 2). Un gros effort fut accompli par la puissance coloniale dans les années cinquante où la découverte du pérrole accéléra la construction de pistes, sommairement équipées dans un premier temps, pour les besoins des pétroliers. Ultérieurement, de véritables aéroports furent crés: Hassi-Messaond et In-Amémas notamment. De nombreuses oasis ont été également désenclavées par la réalisation d'une piste d'atrerrissage (Tindouf, Timimoun, El-Meniaa, Touggourt...), sans compter les besoins propres à l'armée française, que ce soit ponr la lutte contre les combattants de l'Ammée de libération nationale (ALN) ou pour d'autres besoins (expérimentaux en particulier: Tamanghasset, Reggane, Beni- 


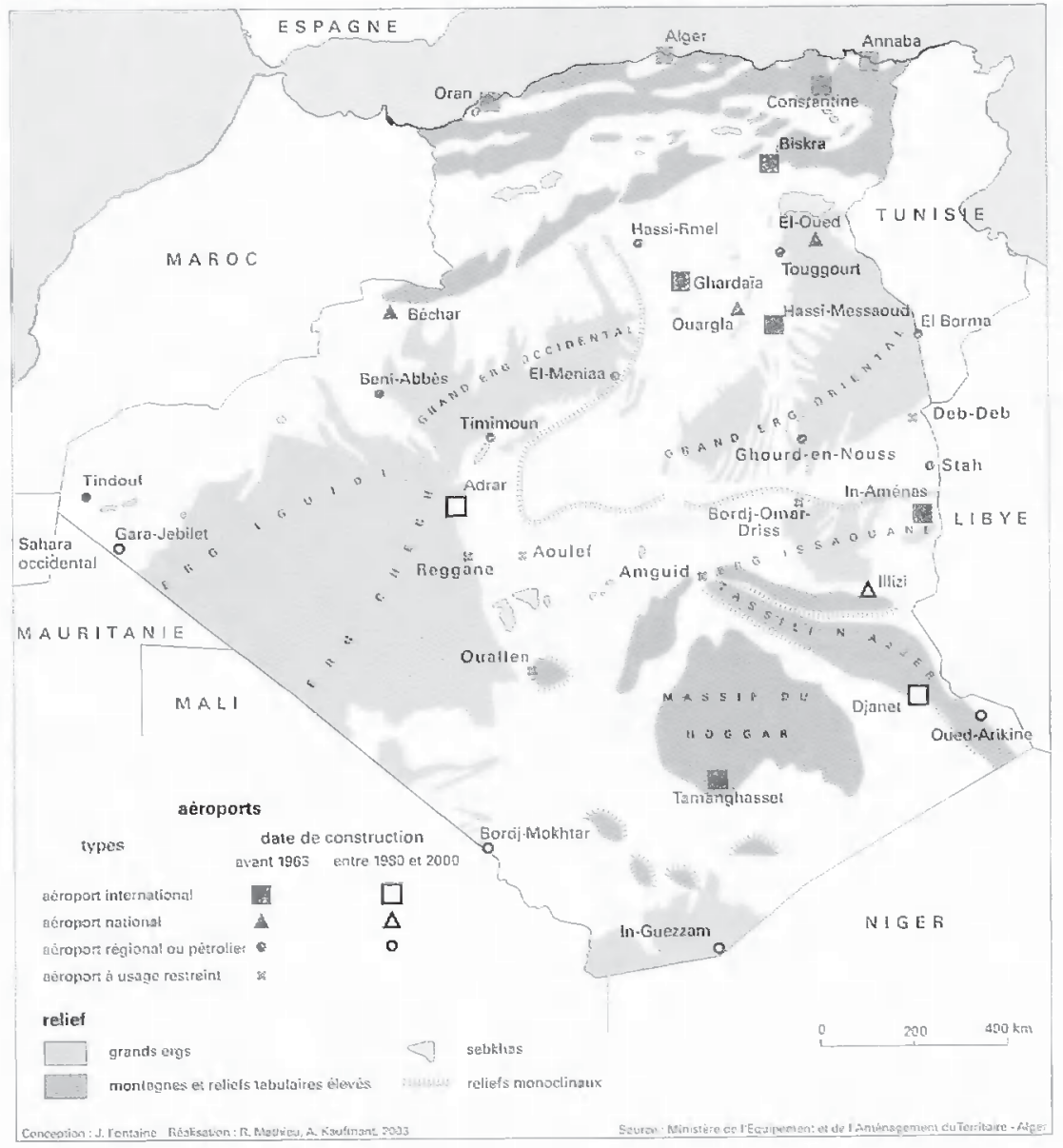

Fig. 2 Les aéroports du Sahara algérien.

Algerian Sahara airports.

Abbès). De ce fait, au lendemain de l'indépendance, le Sahara algérien disposait de plus d'une quinzane d'aéroports et d'une demi-douzaine de pistes «à usage restreint", parfois perdues dans un environnement presque exclusivement minéral (Amguid, Ouallen...). Les autorités algériemnes n'ont donc pas créé de nouveaux aéroports (à l'exception d'In-Salah, 1967). Elles utilisent, généralement modestement, les infiastructures existantes, au moins jusque vers 1975 (sauf dans les zones pétrolières). Au-delà de cette date, un effort important fut fait, aussi bien pour la création d'aćroports remplaçant des infrastructures inadaptées (1)janet, Adrar) ou désenclavant des régions isolées (IIlizi, In-Guezzan, Bordj-Mokhtar) que ponr le développement du réseau: renforcement des liaisons avec Alger (et secondairement avec Oran, Constantine et Annaba) er surtout développenent des 


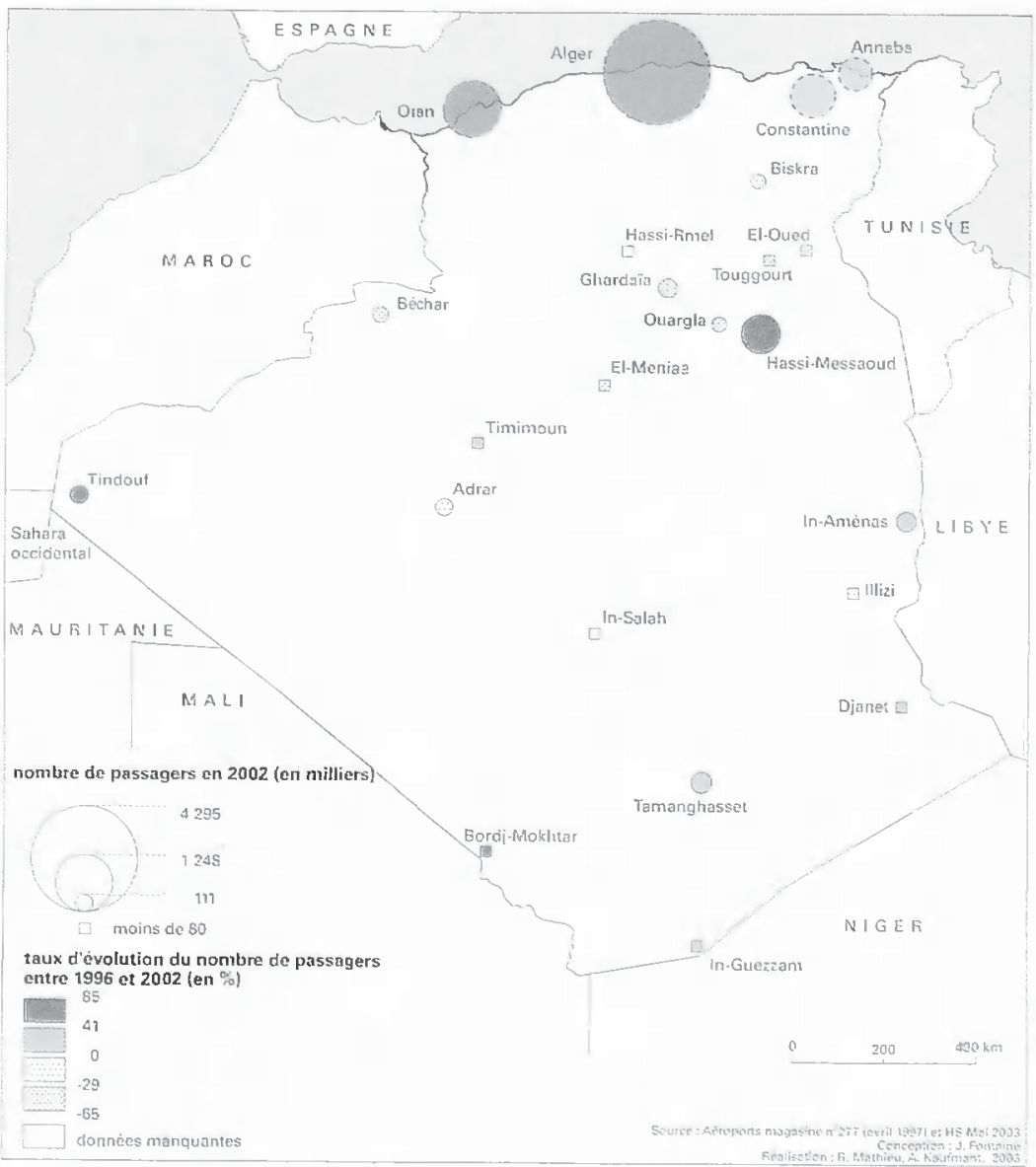

Fig. 3 Le trafic des aéroports du Sahara algérien. Algerian Sahara airports traftic flow:

relations intra saharienaes autour de Ghardaïa qui est une vérimable plaque tournante, mais aussi autour de Bechar, Ouargla, Adrar et même Tamanghasset.

Aujourd'hui sur la trentaine d'aéroports salhariens, sepr peuvent recevoir des vols internationaux (fig. 2). Seuls cependant Biskra, Hassi-Messaond et Tamanghasset sont reliés par des vols réguliers à l'étranger. Les autres villes n'ont que des liaisons à la demande. Elles se développent en particulier avec les acroports du Grand Sud. Cependant, l'essenciel du tratic reste domestique pour tous les aéroports sahariens, les relations avec Alger étant très largement majoritaires. Aujourd'hui, Hassi-Messaoud (fig. 3) est la principale plate-forme aéroportuaire du Sahara. Avee 590000 passagers en 2002, la ville pétrolière dispose du $4^{c}$ aéroport algéricn, avamt Anmaba. La relance 
du secteur des hydrocarbures a entrainé une forte croissance de son trafic depuis 1996. Les autres aéroports sahariens n'ont pas une importance comparable, aucun n'atteint un trafic annuel de 200000 persomes et, bien souvent, ce trafic est en régression plus ou moins prononcée depuis quelques années. La crise algérienne des années 1990 est également passée par là et la relance a touché d’abord le Nord, en particulicr le trafic international. En fait, elle concerne peu le Sahara, en dehors des zones pétrolières et du Grand Sud où le redémarrage du tourisme, encore modeste, explique le développenent du trafic à Tamanghasset et à Djanet.

Le Sahara dispose d'un réseau d'infrastructures norable, en voic d'amélioration permanente (développements du réseau routier, remise en état de la voie ferrée de Bechar programmée, réfection complète de l'aéroport d'Hassi-Messaoud à partir de lautomne $2003 \ldots$. . Ce réscau ne peut que faroriser la mobilité des hommes qui, même à l'époque des pistes et des carałancs, était déjà importante.

\section{Infrastructures, flux migratoires et sous-régions intra-sahariennes}

Les infrastructures mises en place facilitent les déplacements. Elles tendent à organiser des sous-régions, attractives ou répulsives, comme le montre l'étude des migrations (Kouzmine, 2003). Elles mettent en relation des zones parfois très éloignées, parfois très proches aussi. Elles canalisent des flux de départ vers l'Algérie du Nord ou en provenant. L'ćtude des migrations pemet une typologie des régions sahariennes en relation arec leurs infrastructures (fig. 4).

Les régions attractives - essentiellement situées dans le Bas-Sahara avec au premier rang la région de Ouargla/Iassi-Messaoud - reçoivent des flux très variés, aussi bien en migrations définitives qu'en migrations de maind'ouvre. Les flux intra sahariens, provenant principalement du Souf et de l'Oued-Righ, sont dominants. La qualité et la densité du réseau routier favorisent grandement ces migrations. Les flux venant de l'Algérie du Nord ont une importance certaine. L'Aurès envoie nombre de ses hommes, soit provisoirement soit défnitivement, vers les Ziban er le Sahara pétrolier. La région algéroise foumit hon nombre de cadres installés à Ouargla et HassiMessaud, alors que des milliers de travailleurs proviennent de l'agglomération algéroise ou de Grande Kabylie. Ainsi se poursuit une tradition migratoire, autrefois tournée vers le nord de la Méditerranée, orientée désomais rers les chantiers d'hydrocarbures du Sahara. L'importance de lacroport d'Hassi-Messaoud, celle des navertes d'autocar entre les chantiers d'hydrocarbures et la région algéroise ne s'expliquent pas autrement. Les régions d'ln-Aménas-Illizi et celle de I aghouat Hassi-R"nel comaissent le mêne type de fonctionnement que la région Ouargla/l-Gassi-Messaoud, mais avec des flux moindres et des modalités différentes (plus grande importance de lavion pour la première, de l'autocar pour la seconde). 


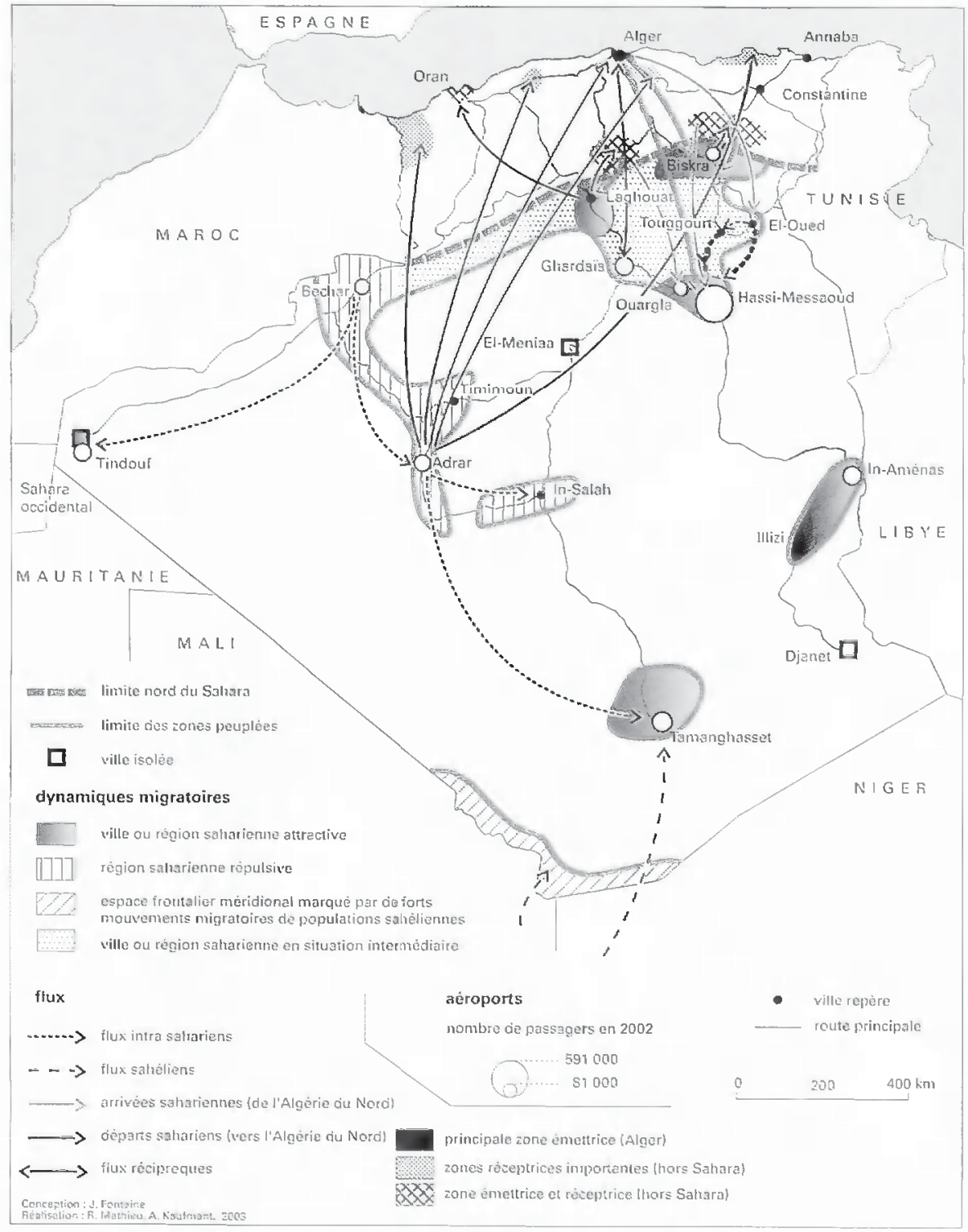

Fig. 4 Organisation spatiale du Sahara algérien: infrastuctures et flux migratoires. A/gerian Sahara spatial organization: networks and migratory flows.

Les autres régions atuatives reçoivent principalenneni des flux sahariens internes ou externes; c'est le cas de 'Tamanghasset et de la zone frontalière méridionale sur le trajet d'importants flux sahéliens, voire africains. 
Les régions répulsives de l'Ouest saharien (Touat, Saoma et Gourara principalement), du fait de l'atonie de leur économie, sont à l'origine de deux types de mouvements. On recense des départs généralement définitifs vers le 'Tell (Skikda, Jijel, Clılef, région algéroise, Tlencen). On observe aussi des migrations - définitives ou de main-d'ouve - vers diverses zones sahariemnes. Ce qui est remarquable dans les deux cas, c'est l'absence de destination privilégice.

Enfin, le piémont saharien (en dehors des Ziban et de Laghouat) apparaît comme une zone atone. Léconomic, liée à la steppe, y reste fondamentalemenr pastorale. L'attractivité des rares villes y est fible eat les mouvements migratoires peu acrifs.

\section{Conclusion: Infrastructures et nouvelles oasis}

Si pendant longtemps le Sahara a pu donner l'image d'un milieu isolé, en marge - encore qu'il ait été traversé par les caravanes pendant des centaines d'années - ce n'est plus du rout le cas aujourd'hui. Par ses activités économiques (principalement pétrolières et gazières nais aussi agricoles), ses infrastructures (notamment routières er aériennes), et les migrations de ses hommes, il est totalement intégré à l'État-Nation algérien.

En un demi-siècle, un effort considérable a été fait pour les infrastrucnures $(8000 \mathrm{~km}$ de routes et une trentaine d'aćroports dont une vingtaine sont desservis par des vols réguliers), ce qui a favorisé des migracions devenues de plus en plus intenses, ainsi que l'a montrés le dernier recensement (RGIPH 1998). Que ce soit pour les migrations détinitives on les flux de main d'oeuve, les Sahariens se déplacent plus que les Algériens diu Nord.

Ces migrations, qu'elles soient nationales ou internationales, ne sont pas saus influence sur le réseau ubain du Sahara algérien. Certaines villes en Lirent plus profit que d'autres; l'utilisation des modes de transport modernes fait que certaines vieilles oasis étnpes (El-Menia, Timimoun, Touggourt...) voient leur rôle décliner alors que d'autres ont une importance sans cesse grandissante: Parmi celles-ci, cirons le binôme Ouargla/HassiMessaoud. C'est le premier centre récepteur de migrants définitifs et de main-d'ouvre, au niveau national. D'autres villes combinent fonction nationale et internationale. A ce titre, le cas de Tamanghasser est sans doute le plus caractéristique. Sa promotion voulue par le pouvoir algérien a été considérablement renforcée par l'arrivée de dizanes de milliers d'jmmigrants africains pour qui, dans la plupart des cas, la capitale du Hoggar n’est qu'une étape vers le Nord. Tn-Salah. Adrar et Ghardaïa sont généralement les ćapes suivantes, avant le passage de la frontière marocaine et l'Europe.

Ainsi, sous la triple poussée de la volonté de contróle et d'organisation du territoire par l'État algérien, des mouvements migratoires nationaux er des migrations internationales, le réseau urbain saharien ne cesse d'ćvoluer. 
Les villes-relais se multiplient et les oasis jouent maintenant un rôle de plaques toumantes, de pôles de redistribution des hommes ou d'accumulation (daus le cas des zones frontalières), le tout daus un réseau de plus en plus maillé. Au coeur de ce réseau un axe méridien semble aujourd'hui privilégié, c'est celui qui relie Alger à Agadez par Ghardaia et Tamanghasset.

\author{
THEAMA, Université de Franche-Comté \\ 32, rue Mégevand \\ 25030 Besancon cedex \\ jacques fontaine@univ-fcomte.ir
}

\title{
Bibliographie
}

Aéroports magazine (1997), n 27\%, avril, Paris.

Aéroports magazine (2003). HS mai, Paris.

Bisson I. (1996), "Le Sahara entle exploration et indépendances - Université de Tours", URBAMA (URA 365) Les cahiers d'URGAMA, n 12 et Université de Franche-Connté, Les Cahiers de /'Universilé Ouverte n ${ }^{\circ}$, p. 45-62.

Bisson ). (2003), Mythes et réalités d'un désent convoité: le Sahara, Paris, L'Harmattan, 480 p.

Blin L. (1990), L'Algérie du Sahara du Sahel, Paris, L"Harmattan, 501 p.

El Watan (25 août 2003), Avis d'appels d'offre, Alger.

Kouzmine Y. (2003), L'espace saharien algérien, dynamiques démographiques el migratoires, mémoire de maîtrise, Besançon, 243 p.

ONS (Office National des Statistiques) (2001), DS n³15 - Migrations internes inter-wilaya, à travers les résultats exhaustís du RGPH 1998-Âlger.

Pliez O. (2002), "Vieux réseaux el nouvelles circulations entre les deux rives du Sahara", Méditeranée, n $3-4$, p. 31-40. 